\title{
First-line treatment and survival of newly diagnosed primary plasma cell leukemia patients in the Netherlands: a population-based study, 1989- 2018
}

\author{
Mirian Brink (1)', Otto Visser ${ }^{2}$, Sonja Zweegman ${ }^{3}$, Pieter Sonneveld ${ }^{4}$, Annemiek Broyl ${ }^{4}$, Niels W.C.J. van de Donk ${ }^{3}$ and \\ Avinash G. Dinmohamed (1,3,5
}

Dear Editor,

Primary plasma cell leukemia (pPCL) is the rarest and most aggressive entity within the spectrum of plasma cell dyscrasias, accounting for nearly $2 \%$ of all plasma cell dyscrasias ${ }^{1}$. Outcomes in PPCL have generally been grim with a median overall survival (OS) of $<12$ months $^{2-4}$. Although currently there is a paucity of data on outcome in PPCL-which are mostly derived from retrospective studies-these data provided hints that the introduction of autologous stem cell transplantation (autoSCT) as well as proteasome-inhibitor (PI)-based and immunomodulatory (IMID)-based therapies have benefits for patients with $\mathrm{pPCL}^{5-8}$. Indeed, the most far-reaching populationbased study-which included 445 pPCL patients diagnosed in the US during 1973-2009-demonstrated that survival in PPCL improved notably since 2006, especially for elderly patients ${ }^{4}$. This improvement was likely a consequence of the availability of PI-based and IMiDbased therapies for first-line treatment in the US from 2006 onwards. However, the improvement could not be directly linked to changing treatment practices, as information on treatment lacked in that study.

Given the scarcity of clinical and population-based studies in PPCL, we conducted a population-based study to assess trends in first-line therapy and survival among

\footnotetext{
Correspondence: Mirian Brink (m.brink@iknl.nl)

${ }^{1}$ Department of Research and Development, Netherlands Comprehensive Cancer Organisation (IKNL), Utrecht, The Netherlands

2Department of Registration, Netherlands Comprehensive Cancer Organisation (IKNL), Utrecht, The Netherlands

Full list of author information is available at the end of the article

These authors jointly supervised this work: Niels W.C.J. van de Donk, Avinash G. Dinmohamed
}

patients with pPCL diagnosed during a 30 -year period in the Netherlands.

We identified all pPCL patients diagnosed between 1989 and 2018 from the nationwide Netherlands Cancer Registry (NCR), which was founded in $1989^{9,10}$. The primary endpoint was OS, defined as the time from diagnosis until death from any cause. Patients alive were censored on February 1, 2020. The log-rank test was used to test for differences between survival distributions. Multivariable Cox regression was applied to estimate the adjusted risk of mortality. Information on primary therapy was available for each patient in the NCR. Calendar period analyses (1989-2000, 2001-2007, and 2008-2018) were conducted to assess trends in primary therapy and OS according to age at diagnosis ( $\leq 65$ and $\geq 66$ years). The calendar periods were defined accordingly to changing treatment practices in multiple myeloma (MM) in the Netherlands. The full methods of our study are provided in the Supplemental. The Privacy Review Board of the NCR approved the use of anonymous data for this study.

The baseline characteristics of 226 patients with pPCL (median age, 66 years; range, 34-91 years; 52\% males) included in this study are presented in Supplemental Table 1. One patient diagnosed at autopsy was excluded.

As shown in Fig. 1A, first-line treatment without SCT was commonly applied among patients aged $\leq 65$ years in the periods 1989-2000 (65\%) and 2001-2007 (59\%). Thereafter, the incorporation of SCT into the first-line treatment increased markedly, namely from 23 to $60 \%$ between $1989-2000$ and 2008-2018 $(P=0.004)$. As for patients aged $\geq 66$ years, the proportion of patients who did not start with treatment was higher as compared to 


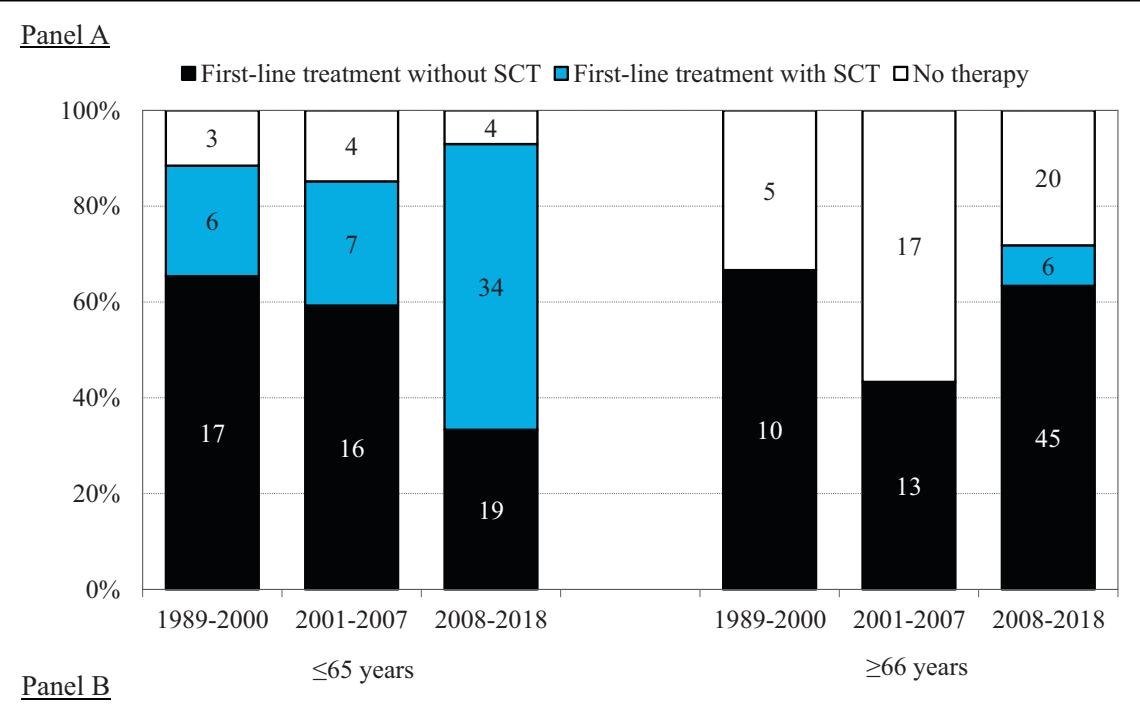

$\square \mathrm{VCD} \square \mathrm{VRD} \square \mathrm{VTD} \square \mathrm{PAD} \square \mathrm{KRD} \square \mathrm{MPV} \square \mathrm{RD} \square \mathrm{VD} \square \mathrm{MP} \square$ no therapy

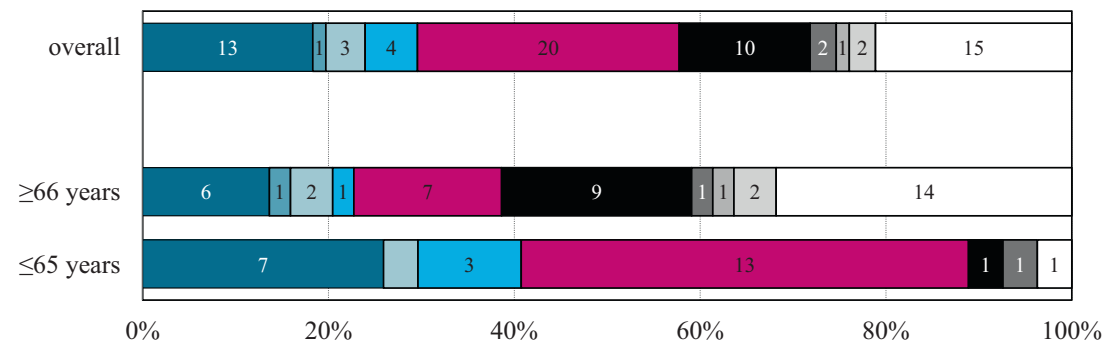

Fig. 1 First-line treatment of patients with PPCL in the Netherlands. The absolute numbers of patients are depicted in the bars. A The first-line treatment for patients diagnosed with PPCL between 1989 and 2018 according to age at diagnosis and calendar period of diagnosis. B The information on the exact therapeutic regimen for diagnosed patients with PPCL between 2014 and 2018 according to age at diagnosis. Of note, patients who received KRD were treated within the setting of a clinical trial. PPCL primary plasma cell leukemia, SCT stem cell transplantation, bortezomib-dexamethasone (VD), VD with cyclophosphamide (VCD), VD with lenalidomide (VRD), VD with thalidomide (VTD), lenalidomidedexamethasone (RD), RD with carfilzomib (KRD), VD with doxorubicin (PAD), melphalan-prednisone (MP), and MP with bortezomib (MPV).

patients aged $\leq 65$ years $(P<0.001)$. Although the proportion of patients aged $\geq 66$ years not receiving therapy is decreasing over time, a substantial number of these patients still did not receive any therapy in the most recent calendar period $(28 \%$ and $49 \%$ in $2008-2018$ and 1989-2007, respectively; $P=0.030$ ). However, noteworthy is the application of SCT among patients aged $\geq 66$ in the most recent calendar period (8\%; median age, 68 years; range, 66-69 years).

Detailed data on baseline characteristics of 71 patients diagnosed in 2014-2018 are presented in Supplemental Table 2. Of these patients, the majority was treated with a PI-based regimen (58\%; Fig. 1B). Twenty-two patients received an SCT as part of their first-line treatment, of whom nine received an autoSCT, eight the tandem of autoSCT and allogeneic SCT, and five a tandem autoSCT.

Overall, the median follow-up was 9.3 months (range, 0.03-198.3 months), whereas it was 52.0 months (range, 13.7-198.3 months) for patients alive at the end of follow-up. For all patients, median OS across the three calendar periods was 8.8, 5.0, and 14.4 months, respectively $(P<0.001$; Supplemental Fig. 1$)$. The corresponding estimates for patients aged $\leq 65$ and $\geq 66$ were $12.2,13.8$, and 28.4 months $(P=0.002)$, and $2.0,2.4$, and 6.4 months $(P=$ 0.009), respectively (Fig. 2). Although OS improved over time, early mortality within 6 months after diagnosis remained high over time for both age groups. Among patients aged $\leq 65$, the proportion of early mortality was $31 \%, 33 \%$, and $25 \%$ for the three consecutive calendar periods $(P=0.667)$. The corresponding proportions for patients aged $\geq 66$ years were $73 \%, 70 \%$, and $49 \%(P=0.067)$. The projected OS at 6 months and 1,2, and 5 years postdiagnosis according to age at diagnosis and calendar period of diagnosis are presented in Fig. 2.

When simultaneously adjusted for sex, age at diagnosis, and calendar period of diagnosis, patients diagnosed during 2008-2018 had a lower risk of mortality (adjusted hazard ratio $[\mathrm{HR}], 0.59 ; 95 \%$ confidence interval $[\mathrm{CI}]$ 


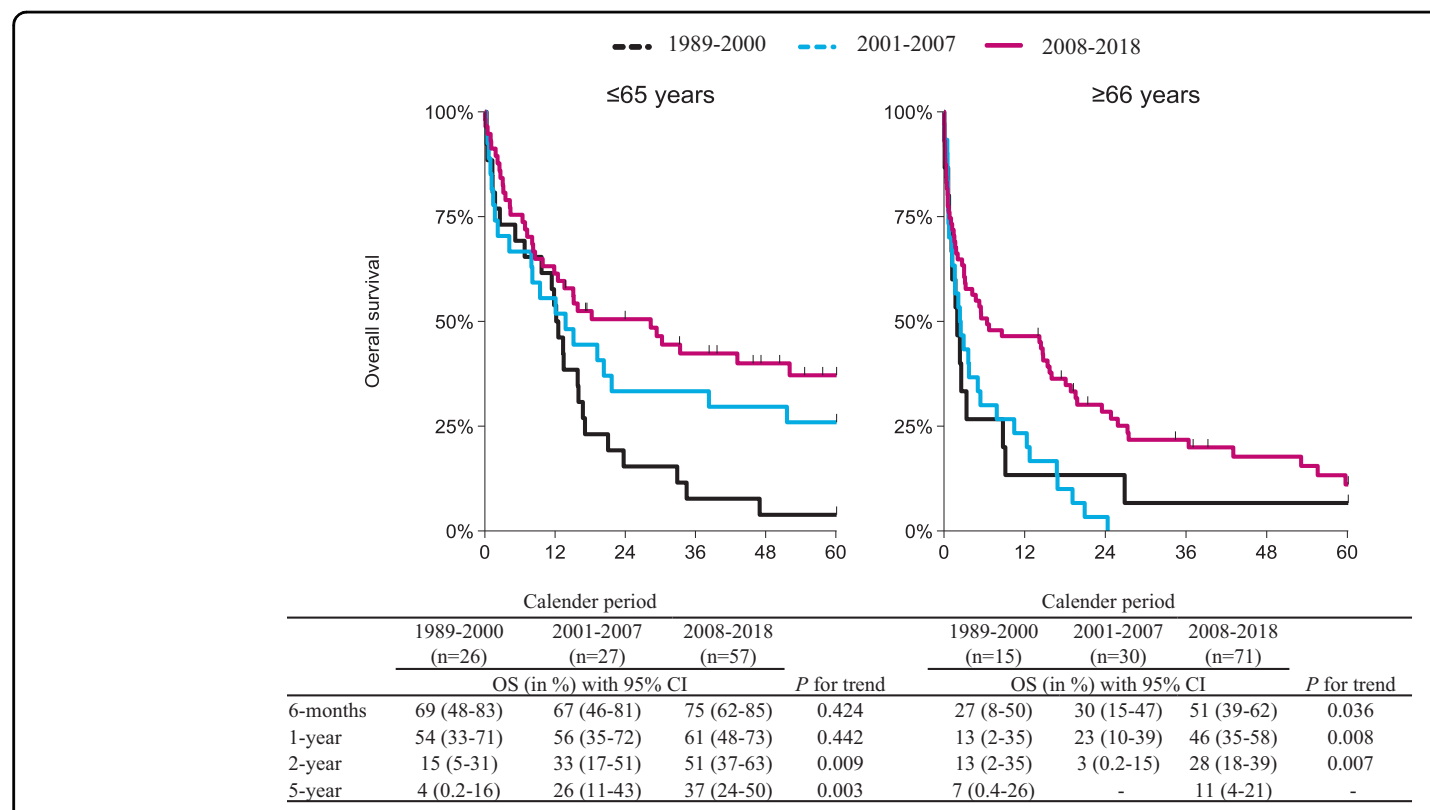

Fig. 2 Overall survival (OS) of patients with pPCL in the Netherlands, 1989-2018. OS is stratified by calendar period of diagnosis and shown for the following age categories: $\leq 65$ years (left panel), and $\geq 66$ years (right panel). The $P$-value of the log-rank test for trend is indicated in the Figure.

$0.42-0.84 ; P=0.003)$, as compared to patients diagnosed before 2007. Furthermore, patients aged $\geq 66$ years had a higher risk of mortality (adjusted HR, 2.19; 95\% CI $1.63-2.94 ; P<0.001)$, as compared to patients aged $\leq 65$ years (Supplemental Table 3). However, when additionally adjusted for first-line treatment, the prognostic effects of calendar period and age lost statistical significance. This finding suggests that the broader application of therapy, especially the application of SCT, contributed to the improved survival over time, and this was irrespective of age (Supplemental Table 3).

In this comprehensive, nationwide, population-based study, the survival of pPCL patients improved over time. However, early mortality remains high, which is probably related to both disease-related and therapy-related complications. Our study is the first to directly link the improvements in survival with changes in first-line therapy over time. Collectively, patients with pPCL likely benefited from the therapeutic advances achieved over the past decades.

Our findings are mostly in keeping with a previous population-based study conducted in the US during $1973-2009^{4}$, and prospective and ${ }^{10}$ retrospective studies ${ }^{11,12}$, including a multicenter study conducted in Greece during $2000-2016^{5}$. However, in our study, the median OS of patients aged $\geq 66$ years diagnosed during 2008-2018 was lower, as compared to the median OS of patients $\geq 66$ years diagnosed during 2006-2009 in the US - possibly due to a delay in the introduction of more effective therapies in the Netherlands compared to the US. The importance of novel agent-based regimens in
pPCL was also shown among 50 pPCL patients diagnosed in Greece during 2000-2016 . That study revealed that OS was significantly higher among patients managed with bortezomib-based regimens and autoSCT, as compared to those managed with more conventional therapies. Furthermore, our results compare less favorably with two relatively small prospective trials, which are the only trials published to date in $\mathrm{pPCL}^{7,8}$. These differences may be related to the use of novel agent-based regimens in all patients enrolled in these clinical trials, as compared to only $58 \%$ of the patients diagnosed in 2014-2018 in our study. Also, the selection of comparatively fit patients fulfilling the strict inclusion criteria of trials-as compared to the inclusion of all pPCL patients identified in population-based registries-may result in differences between the studies. Concerning stem cell transplantation, the OS in transplant-eligible pPCL patients in three large retrospective series ${ }^{6,13,14}$ was superior, as compared to the OS of pPCL patients aged $\leq 65$ years diagnosed in 2014-2018 in our population-based study-of whom 63\% received autoSCT. However, it is unknown which proportion of patients in the retrospective series ${ }^{6,13,14}$ was planned to undergo autoSCT, but eventually did not receive this treatment because of either early progression or early death. This particular selection bias may lead to an overestimation of the effectiveness of autoSCT in pPCL. Taken together, the therapeutic advances achieved in recent decades seem to gradually translate into tangible benefits for pPCL patients at the population level. Nevertheless, the specific design and conduct of prospective intervention studies in $\mathrm{pPCL}$ across different 
lines of therapy are imperative to advance the evidencebased management of pPCL. International collaboration is needed to accomplish such trials.

The main strength of our study includes the use of a nationwide population-based cancer registry with comprehensive data available on first-line treatment. Therefore, changing treatment practices over time could be assessed and directly linked to improvements in outcome. Limitations of our study mainly pertain to the lack of detailed information on first-line treatment throughout most of the study period (i.e., 1989-2013) and potential misclassification of PPCL as MM or vice versa, especially in earlier periods. Despite these limitations, cancer registries remain the standard for cancer surveillance activities.

In summary, the population-level survival of pPCL patients improved significantly over time. Notwithstanding this encouraging finding, survival in PPCL remains unsatisfactory, especially among the elderly. Therefore, the design and conduct of forthcoming prospective intervention studies for patients with PPCL are essential to establish evidence-based treatment recommendations, which, in turn, may further improve the outcome in this patient population.

\section{Acknowledgements}

The authors would like to thank the registration clerks of the Netherlands Cancer Registry (NCR) for their dedicated data collection. The nationwide population-based NCR is maintained and hosted by the Netherlands Comprehensive Cancer Organisation (IKNL).

\section{Author details}

'Department of Research and Development, Netherlands Comprehensive Cancer Organisation (IKNL), Utrecht, The Netherlands. ${ }^{2}$ Department of Registration, Netherlands Comprehensive Cancer Organisation (IKNL), Utrecht, The Netherlands. ${ }^{3}$ Amsterdam UMC, Vrije Universiteit Amsterdam, Department of Hematology, Cancer Center Amsterdam, Amsterdam, The Netherlands. ${ }^{4}$ Department of Hematology, Erasmus MC Cancer Institute, Rotterdam, The Netherlands. ${ }^{5}$ Department of Public Health, Erasmus University Medical Center, Rotterdam, The Netherlands

\section{Author contributions}

A.G.D. designed the study; MB analyzed the data; O.V. collected the data; M.B., A.G.D., and N.vdD. wrote the manuscript with contributions from all authors, who also interpreted the data, and read, commented on, and approved the final version of the manuscript.

\section{Conflict of interest}

N.W.C.J.vdD. has received research support from Janssen Pharmaceuticals, AMGEN, Celgene, Novartis, and BMS and serves in advisory boards for Janssen Pharmaceuticals, AMGEN, Celgene, BMS, Takeda, Roche, Novartis, Bayer, and Servier. P.S. has received research support from Janssen Pharmaceuticals, AMGEN, Celgene, Karyopharm, and BMS and serves in advisory boards for Janssen Pharmaceuticals, AMGEN, Celgene, BMS, and Carsgene. S.Z. serves in advisory boards for Celgene, Janssen Pharmaceuticals, Sanofi, Takeda, Amgen, and has received research support from Janssen Pharmaceuticals, Takeda and Celgene. A.B. serves in advisory boards for Janssen Pharmaceuticals, AMGEN, Celgene, BMS, Takeda. The remaining authors declare no conflict of interest.

\section{Publisher's note}

Springer Nature remains neutral with regard to jurisdictional claims in published maps and institutional affiliations.

\section{Supplementary information}

The online version contains supplementary material available at https://doi. org/10.1038/s41408-021-00415-5.

Received: 11 September 2020 Revised: 23 December 2020 Accepted: 18 January 2021

Published online: 04 February 2021

\section{References}

1. van de Donk, N. W., Lokhorst, H. M., Anderson, K. C. \& Richardson, P. G. How I treat plasma cell leukemia. Blood 120, 2376-2389 (2012).

2. Katodritou, E. et al. Treatment with bortezomib-based regimens improves overall response and predicts for survival in patients with primary or secondary plasma cell leukemia: analysis of the Greek myeloma study group. Am. J. Hematol. 89, 145-150 (2014).

3. Gavriatopoulou, M. et al. European myeloma network recommendations on diagnosis and management of patients with rare plasma cell dyscrasias. Leukemia 32, 1883-1898 (2018).

4. Gonsalves, W. I. et al. Trends in survival of patients with primary plasma cell leukemia: a population-based analysis. Blood 124, 907-912 (2014).

5. Katodritou, E. et al. Real-world data on prognosis and outcome of primary plasma cell leukemia in the era of novel agents: a multicenter national study by the Greek Myeloma Study Group. Blood Cancer J. 8, 31 (2018).

6. Mahindra, A. et al. Hematopoietic cell transplantation for primary plasma cell leukemia: results from the Center for International Blood and Marrow Transplant Research. Leukemia 26, 1091-1097 (2012).

7. Royer, B. et al. Bortezomib, doxorubicin, cyclophosphamide, dexamethasone induction followed by stem cell transplantation for primary plasma cell leukemia: a prospective phase II study of the Intergroupe Francophone $d u$ Myelome. J. Clin. Oncol. 34, 2125-2132 (2016).

8. Musto, P. et al. Lenalidomide and low-dose dexamethasone for newly diagnosed primary plasma cell leukemia. Leukemia 28, 222-225 (2014).

9. Schouten, L. J., Hoppener, P., van den Brandt, P. A., Knottnerus, J. A. \& Jager, J. J. Completeness of cancer registration in Limburg, The Netherlands. Int. J. Epidemiol. 22, 369-376 (1993).

10. Lebovic, D. et al. Clinical outcomes of patients with plasma cell leukemia in the era of novel therapies and hematopoietic stem cell transplantation strategies: a single-institution experience. Clin. Lymphoma Myeloma Leukemia 11, 507-511 (2011).

11. D'Arena, G. et al. Frontline chemotherapy with bortezomib-containing combinations improves response rate and survival in primary plasma cell leukemia: a retrospective study from GIMEMA Multiple Myeloma Working Party. Ann. Oncol. 23, 1499-1502 (2012).

12. Mina, R. et al. Survival outcomes of patients with primary plasma cell leukemia (pPCL) treated with novel agents. Cancer 125, 416-423 (2019).

13. Drake, M. B. et al. Primary plasma cell leukemia and autologous stem cell transplantation. Haematologica 95, 804-809 (2010).

14. Dhakal, B. et al. Hematopoietic cell transplantation utilization and outcomes for primary plasma cell leukemia in the current era. Leukemia 34, 3338-3347 (2020). 